\title{
Contents
}

\section{General Theory and Applications}

to Unstable Particles

Robert Alicki ....................................... 1

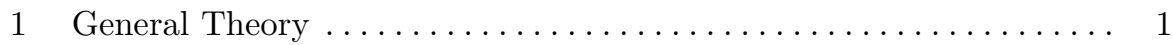

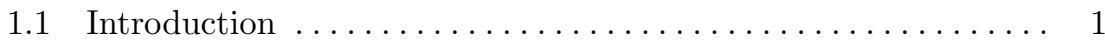

1.2 Completely Positive Dynamical Semigroups .............. 2

1.2.1 Reduced Dynamics ........................ 2

1.2.2 Completely Positive Maps ................... 3

1.2.3 Generalized H-theorem ................... 5

1.2.4 Generators of Quantum Dynamical Semigroups ....... 7

1.2.5 How to Construct Generators? ................. 9

1.3 Hamiltonian Models and Markovian Approximation .......... 10

1.3.1 Generalized Master Equation ................... 10

1.3.2 Weak Coupling Limit . . . . . . . . . . . . . . . . . 11

1.3.3 Low Density Limit ..................... 14

1.3.4 Heat Bath, Detailed Balance and Return to Equilibrium 16

1.3.5 Singular Coupling Limit ..................... 18

1.4 Extensions of the Formalism ..................... 19

1.4.1 Nonconservative Dynamical Semigroups ............ 19

1.4.2 Time-dependent Generators .................. 20

1.4.3 Nonlinear Quantum Dynamical Semigroups .......... 21

1.4.4 Discrete Quantum Boltzmann Equation ............. 22

1.4.5 Nonlinear Schrödinger Equation ................ 23

1.5 A System of $N$ Two-level Atoms . ................... 24

1.5.1 The Hamiltonian of the System . . . . . . . . . . . . . 24

1.5.2 The Markovian Master Equation .............. 25

1.5.3 Return to Equilibrium and Superradiance ........... 27

2 Quantum Dynamical Semigroups

for Unstable Particles . . . . . . . . . . . . . . . . . . . . . . 29

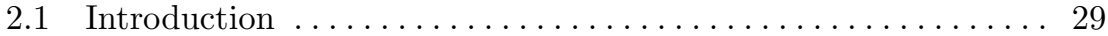

2.2 Damped and Pumped Quantum Harmonic Oscillator ........ 30 
2.2.1 Derivation of the Markovian Master Equation ....... . . 30

2.2.2 Birth and Death Process, Kinetic Equation .......... . 31

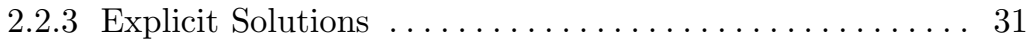

2.3 Models of Unstable Particles . . . . . . . . . . . . . . . 32

2.3.1 Fock Spaces and Quantum Fields.............. 32

2.3.2 Construction of Markovian Master Equation ......... . 34

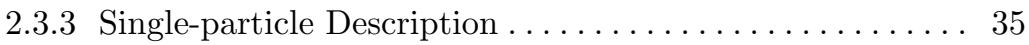

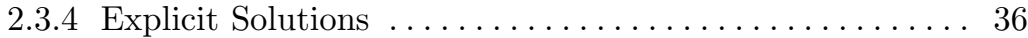

2.3.5 Hamiltonian Models of Unstable Particles ... . . . . . . 38

2.3.6 Relativistic Unstable Particles . . . . . . . . . . . . . 40 Appendix

A.1 Banach Spaces $\mathcal{B}(\mathcal{H})$ and $\mathcal{T}(\mathcal{H}) \ldots \ldots \ldots \ldots \ldots \ldots \ldots \ldots$

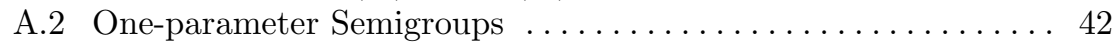

A.3 Quantum Correlation Functions ................... 44

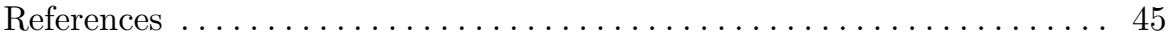

\section{N-Level Systems and Applications to Spectroscopy}

Karl Lendi .................................. 47

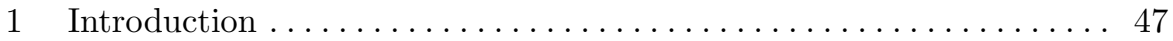

2 General Structure of Quantum Markovian Master Equations

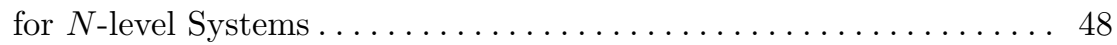

2.1 The Kossakowski-Generator of Infinitesimal Time-evolution . . 48

2.2 Positive-semidefiniteness of the Relaxation Matrix . . . . . . . . . 49

2.3 Complete Orthonormal Matrix Sets . . . . . . . . . . . . . . . . 50

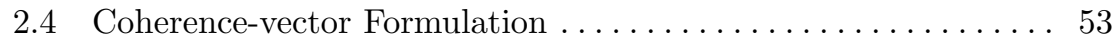

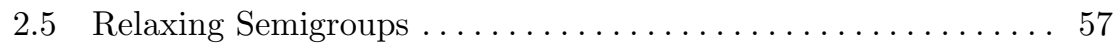

3 Two-level Systems: Generalized Magnetic

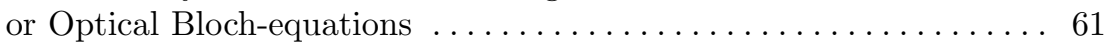

3.1 Details of the Full Relaxation Equations for Static External Fields . . . . . . . . . . . . . . . . . 61

3.2 Alternating External Fields and Constant Relaxation. . . . . . . . . 64

3.3 Modified Lineshapes and Free Induction Decay . . . . . . . . . . 66

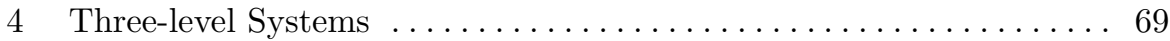

4.1 General Equations . . . . . . . . . . . . . . . . . . . . . . . . 69

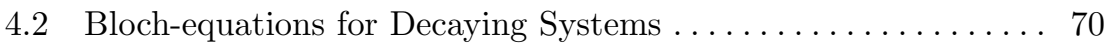

5 Comparison with Common Versions

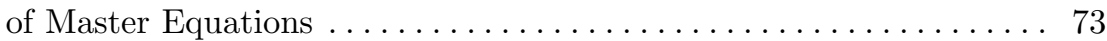

5.1 General Considerations . . . . . . . . . . . . . . . . . 73

5.2 Equations for Spontaneous Emission . . . . . . . . . . . . 74

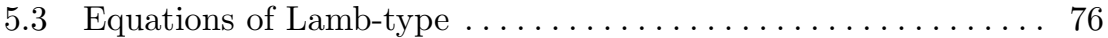

6 Open Quantum Systems with Non-constant Relaxation in Time-dependent External Fields . . . . . . . . . . . . . . . 77

6.1 Modifications of the Original Semigroup Generator . . . . . . . . . 77

6.2 A Model with Field-Strength-dependent Relaxation . . . . . . . . . 79 
7 Determination of Relaxation Parameters

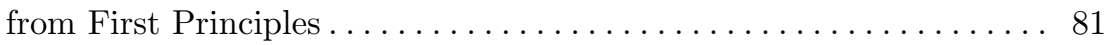

7.1 Relationship between Kossakowski- and Davies-generators . . . 81

7.2 A Model for Spin-relaxation by Spin-waves . . . . . . . . . . . . . 85

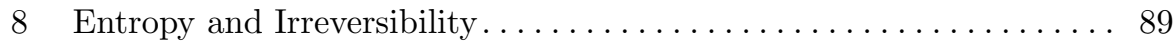

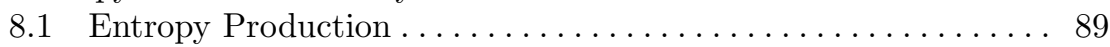

8.2 Measure of Irreversibility $\ldots \ldots \ldots \ldots \ldots \ldots \ldots \ldots \ldots$

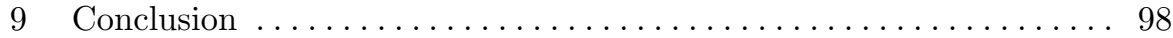

Appendix

A.1 Generators and Structure Constants for $\mathrm{SU}(N), N=2,3,4 \ldots 99$

A.2 Eigenvalues of the General Two-level Evolution Matrix . . . . . . 102

A.3 Elements of the Time-dependent Two-level Evolution Matrix . . 104

References .................................... 104

\section{Recent Developments}

Robert Alicki and Karl Lendi . . . . . . . . . . . . . . . . . . . . . 109

1 Complete Positivity, Entanglement and Decoherence . . . . . . . . . . . 109

2 Unbounded Generators and Stochastic Equations . . . . . . . . . . . 110

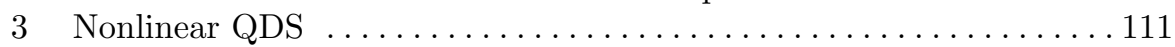

4 Geometry of States and Symmetries of Generators . . . . . . . . . . 111

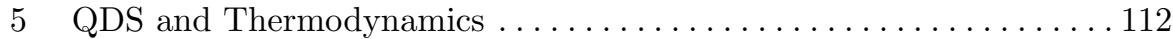

6 Applications in Atomic and Molecular Physics . . . . . . . . . . . . 113

7 Beyond a Markovian Approximation . . . . . . . . . . . . . . . 114

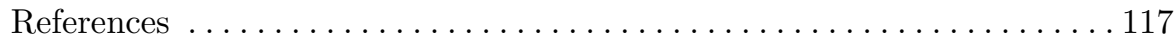

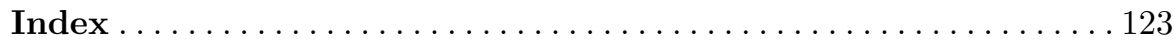

